\title{
Estrogen Causes Ultrastructural Changes of Fibrin Networks During the Menstrual Cycle: A Qualitative Investigation
}

\author{
ALBE C. SWANEPOEL, ${ }^{1 *}$ BAREND G. LINDEQUE, ${ }^{2}$ PAUL J. SWART, ${ }^{2}$ ZEELHA ABDOOL, ${ }^{2}$ AND ETHERESIA \\ PRETORIUS $^{1}$ \\ ${ }^{1}$ Department of Physiology, School of Medicine, Faculty of Health Sciences, University of Pretoria, Pretoria, South Africa \\ ${ }^{2}$ Department of Obstetrics and Gynaecology, Faculty of Health Sciences, School of Medicine, University of Pretoria, \\ Pretoria, South Africa
}

${ }^{*}$ Correspondence to: Albe C. Swanepoel; Department of Physiology, Faculty of Health Sciences, University of Pretoria, Private Bag x323, Arcadia, Pretoria 0007, South Africa.

E-mail: albe.swanepoel@up.ac.za

KEYWORDS menstrual cycle; fibrin network; estrogen; morphology; electron microscopy

\begin{abstract}
Introduction: Hormonal fluctuations may influence fibrin structure. During the menstrual cycle, plasma fibrinogen levels change, mainly due to the variations of estrogen. Throughout the menstrual cycle estrogen levels peak twice, first during the mid-follicular phase and then a lower second peak during the luteal phase. Materials and methods: In order to investigate the possible changes in the fibrin network throughout the menstrual cycle, the fibrin network ultrastructure of six healthy female participants were studied at different intervals in the menstrual cycle where differences in estrogen levels are prevalent. Blood plasma smears were prepared for scanning and transmission electron microscopy analysis. Results: The external and internal structure of the fibrin fibers showed different morphologies throughout the menstrual cycle. The fibrin fibers were smooth during days $1-5$. However, during days $12-14$ of the menstrual cycle the fibrin fiber morphology started to change, becoming less smooth. During the luteal phase of the cycle (days 20-25), the network appears sticky, where the minor, thin fibers are more prominent between the thick fibers when compared to the menstrual phase. Conclusion: The two estrogen peaks of the menstrual cycle coincide with the changes seen in the current qualitative research, where the fibrin morphology changes during the same time as the estrogen peaks occur. Purified fibrinogen confirmed that it is indeed estrogen that causes the altered fibrin network morphology. This research is the first to show ultrastructural changes in fibrin fiber morphology resulting from estrogen changes during the menstrual cycle.
\end{abstract}

\section{INTRODUCTION}

Platelets and fibrin play an important role in the regular coagulation process where they are involved in the maintenance of haemostasis (Herd and Page, 1994). Fibrinogen is the major plasma protein coagulation factor and these fibrinogen molecules assemble to form a clot or thrombus, consisting of a fibrin fiber network. The ultrastructure of this network may change, mainly due to kinetic and modulating factors present in plasma. Also, hormonal fluctuations may influence fibrin structure. An example of this is during the menstrual cycle, when plasma fibrinogen levels change, mainly due to the variations of estrogen (Bailey et al., 2009; Kadir et al., 1999; Lowe et al., 2004); causing an altered fibrin assembly. Estrogen not only alters procoagulant protein expression (Butenas and Mann, 2002; Kadir et al., 1999; Leng et al., 2004; Lowe et al., 2004 ); but differences in platelet function and thrombosis activity have also been noted (Bailey et al., 2009; Liao et al., 2001; Suzuki et al., 2009).

Functionally, female platelet isolates, though capable of binding more fibrinogen and displaying a greater maximal aggregation extent than male platelet isolates (Bailey et al., 2009; Leng et al., 2004), do not aggregate as quickly as the larger number of male platelets does. Thus, to some degree females are rendered less susceptible to thrombosis. Estrogen is known to markedly decrease the risk of cardiovascular up to 50\%, showing the benefit of estrogen (Wong et al., 2008). Gebara et al. showed estrogen to play a cardio-protective role by increasing the fibrinolytic potential (van der Spuy and Pretorius, 2012). Estrogen treat-ment has been shown to shift the haemostatic balance toward a hypercoagulable state (Knobil et al., 1998).

Throughout the female reproductive lifetime, estrogen plays a fundamental role, as gametes are liberated at specific intervals while estrogen and progesterone along with other sex steroids prepare her body for reproduction. The menstrual cycle is the period characterized by ovum maturation, the release of the ovum 
where after it passes through the fallopian tubes to enter the uterine lumen. The endometrium undergoes changes to accommodate a fertilized ovum to sustain pregnancy. These changes are brought about by ovarian hormonal secretions. If fertilization does not occur ovarian secretions cause the endometrium to slough and menstruation commences. Menstrual flow also referred to as menses, marks the first day of the menstrual cycle (Silverthorn et al., 2009). If fertilization does take place, estrogen and progesterone both play critical roles in maintaining the pregnancy.

Two additional cycles can be distinguished within the menstrual cycle. The ovarian cycle relates to the alterations observed in the follicles of the ovary while the uterine cycle concerns the changes in the lining of the endometrium. The ovarian cycle is divided into a further three phases starting with the follicular phase characterized by the growth of the ovarian follicle, followed by ovulation or the release of the matured ovum and ending with the luteal or postovulatory phase. The follicular phase is characterized by elevated levels of the hormone estrogen while the luteal phase is dominated by elevated concentrations of progesterone. Both these essential steroid hormones are secreted by the ovaries (Niswender and Nett, 1994; Silverthorn et al., 2009).

Throughout the menstrual cycle estrogen levels peak twice. The first estrogen peak is reached during the mid-follicular phase (between days 12 and 14 of the cycle) and then rapidly decreases. This estrogen peak triggers LH secretion which in turn leads to ovulation. During the luteal phase, the corpus luteum secretes progesterone and estrogen. General gynaecologic handbooks state that approximately 8-9 days after ovulation (days 20-23 of the menstrual cycle) progesterone levels reach a peak, along with a lower second peak of estrogen (Dighe et al., 2005; Mosesson, 2005).

The question that now arises is whether changes in the particularly the fibrin network are visible at an ultrastructural level, due to the subtle estrogen changes during the menstrual cycle. In order to investigate this question, six healthy female participants were identified and the ultrastructure of their fibrin network studied at different intervals in the menstrual cycle where differences in estrogen levels are prevalent.

\section{MATERIALS AND METHODS}

This study is divided into three parts namely Experiment 1, analyzing the fibrin external ultrastructural differences throughout the menstrual cycle; Experiment 2, analyzing the internal structure of the fibrin network throughout the menstrual cycle; and Experiment 3, which focused on the influence of estradiol on a purified fibrinogen model. The second and third experiments were conducted to confirm results from Experiment 1.

\section{Experiment 1}

Blood Collection. Blood was collected from six healthy, female participants. These individuals were non-smokers, did not have a history of thrombotic disease or used any chronic medication known to interfere with coagulation factors and/platelet function. The participants have never used contraceptive medication neither were they using aspirin or aspirin analogues within $48 \mathrm{~h}$ before sampling. Blood was collected in a citrate tube (containing 0.5 $\mathrm{mL}$ of $3.8 \%$ sodium citrate) as well as a serum gel tube (containing clot activator and gel for serum separation) on three occasions during the menstrual cycle. The three occasions included were the menstrual phase (days 1-5, day 1 with the commencement of menses), the pre-ovulatory phase (days 1214, first estrogen peak) and the luteal phase (days 20-25, progesterone peak and second estrogen peak). A volume of $5 \mathrm{~mL}$ of blood was collected per blood tube. Citrated whole blood was used for morphological analysis while the blood in the gel tube was sent for estradiol concentration analysis. The cycles of all the individuals were 28 days each.

Sample preparation for morphological analysis. The citrated whole blood was centrifuged for $10 \mathrm{~min}$ at $1,000 \mathrm{rpm}$ (maximum $\mathrm{RCF}=17.523 \mathrm{~g} ; 1,250 \mathrm{~g}$ ). The plasma was transferred to a $1.5 \mathrm{~mL}$ eppendorf tube and centrifuged once more for $10 \mathrm{~min}$ at $1,250 \mathrm{~g}$. The platelet poor plasma (PPP) obtained was used to make a fibrin smear on a round glass cover slip for scanning electron microscopy (SEM) analysis and a fibrin clot for transmission electron microscopy (TEM) analysis.

Thrombin (provided by The South African National Blood Services) was added to the PPP. The thrombin was $20 \mathrm{U} / \mathrm{mL}$ and was prepared in biological buffer containing $0.2 \%$ human serum albumin. When thrombin was added to PPP, fibrinogen was converted to fibrin to form an expansive fibrin fiber network.

$10 \mu \mathrm{L}$ of PPP was mixed with $10 \mu \mathrm{L}$ thrombin on a glass cover slip for SEM analysis and the same amounts were mixed in an eppendorf tube for TEM analysis.

Preparation of Samples for SEM. The samples on the cover slips were immediately placed in a sixwell plate on filter paper dampened with PBS to create a humid environment and placed at $37^{\circ} \mathrm{C}$ for $10 \mathrm{~min}$. Following incubation the glass cover slips with the coagula were covered with PBS and placed on a plate shaker for $20 \mathrm{~min}$. This washing process was done to remove any blood proteins trapped within the fibrin network.

After the washing process, the samples were fixated with a $2.5 \%$ gluteraldehyde solution for $30 \mathrm{~min}$. After primary fixation, the samples were rinsed three times in $0.075 \mathrm{M}$ sodium potassium phosphate buffer $(\mathrm{pH}=7.4)$ for $5 \mathrm{~min}$ each. After rinsing, the sample was placed in $1 \%$ osmium tetraoxide $\left(\mathrm{OsO}_{4}\right)$ solution for $30 \mathrm{~min}$. Following secondary fixation, the samples were rinsed once more as described above. The samples were then dehydrated in 30\%, 50\%, 70\%, 90\% and three changes of $100 \%$ ethanol. Procedures were concluded by critical point drying of the samples, mounting the samples and coating it with carbon. The samples were examined with a Zeiss Ultra plus FEG scanning electron microscope.

\section{Experiment 2}

Preparation of Samples for TEM. The fibrin clot samples in the eppendorf tubes were incubated at $37^{\circ} \mathrm{C}$ for 5 min. Following incubation the samples were washed for $20 \mathrm{~min}$. This washing process was done to 
remove any blood proteins trapped within the fibrin network.

The samples were prepared as described in the SEM preparation until after the dehydration step. Following dehydration the samples were infiltrated in a mixture of one part ethanol and one part epoxy resin for $30 \mathrm{~min}$ and then stored in pure epoxy resin for $4 \mathrm{~h}$. The samples were then placed in a rubber mould filled with pure epoxy resin and left to set for 3 days overnight in an oven at $60^{\circ} \mathrm{C}$. Ultra-thin sections of $80-100 \mathrm{~nm}$ were cut with a diamond knife using an ultramicrotome. The sections were contrasted with uranyl acetate for 7 min followed by contrasting with lead citrate for $5 \mathrm{~min}$, after which samples were allowed to dry for a few minutes before examination with the JEOL transmission electron microscope (JEM 2100F). Photomicrographs revealing the internal structures of the fibrin fibers were taken.

For all experiments a blinding procedure between observer and investigator was followed to minimize bias.

Estradiol Concentrations. Serum gel tubes containing whole blood was analyzed for estradiol levels (in pmol/L) by Ampath pathology laboratory support services on the specific days that blood was drawn in the menstrual cycle. Results were supplied in report format indicating estradiol concentrations as well as the ranges for estradiol at different stages during the menstrual cycle.

\section{Experiment 2}

Sample Preparation. Purified fibrinogen, with a concentration of $0.166 \mathrm{mg} / 10 \mathrm{~mL}$, was used to make two smears. Fibrinogen is converted to fibrin by thrombin. (O'Riordan and Higgins, 2003) For the first smear $10 \mu \mathrm{L}$ of fibrinogen was placed on glass cover slip and mixed with $5 \mu \mathrm{L}$ thrombin to make a fibrin smear. For the second smear, to imitate the pre-ovulatory phase of the menstrual cycle, $10 \mu \mathrm{L}$ of fibrinogen, and $5 \mu \mathrm{L}$ estrogen concentration was placed on a glass cover slip and mixed with $5 \mu \mathrm{L}$ thrombin. The estrogen concentration increases to almost $1,200 \mathrm{pmol} / \mathrm{L}$ in the days leading up to ovulation (Giardina et al., 2004). This estrogen increase is the first and highest estrogen peak in the menstrual cycle. A concentration of 1,000 $\mathrm{pmol} / \mathrm{L}$ was therefore used to imitate the pre-ovulatory estrogen peak. Since the estrogen volume was one quarter of the total volume of the fibrinogen, estrogen, and thrombin mixture, the estrogen concentration added to the mixture was $4,000 \mathrm{pmol} / \mathrm{L}$ to make the final concentration of $1,000 \mathrm{pmol} / \mathrm{L}$ in the coagula.

Preparation of Samples for SEM. The samples on the cover slips were immediately placed in a sixwell plate on filter paper dampened with PBS to create a humid environment and placed at $37^{\circ} \mathrm{C}$ for $10 \mathrm{~min}$. Following incubation the glass cover slips with the coagula were covered with PBS and placed on a plate shaker for $20 \mathrm{~min}$. This washing process was done to remove any blood proteins trapped within the fibrin network.

After the washing process, the samples were fixated with a $2.5 \%$ gluteraldehyde solution for $30 \mathrm{~min}$. After primary fixation, the samples were rinsed three times in $0.075 \mathrm{M}$ sodium potassium phosphate buffer $(\mathrm{pH}=7.4)$ for $5 \mathrm{~min}$ each. After rinsing, the sample was placed in $1 \%$ osmium tetraoxide $\left(\mathrm{OsO}_{4}\right)$ solution for $30 \mathrm{~min}$. Following secondary fixation, the samples were rinsed once more as described above. The samples were then dehydrated in $30 \%, 50 \%, 70 \%, 90 \%$, and three changes of $100 \%$ ethanol. Procedures were concluded by critical point drying of the samples, mounting the samples and coating it with carbon. The samples were examined with a Zeiss Ultra plus FEG scanning electron microscope.

\section{RESULTS \\ Experiment 1 and 2}

Morphological Findings. With the addition of thrombin to PPP, an extensive fibrin fiber network forms. This network typically consists of major, thick fibers with a few scattered minor fibers in between the thick fibers. During the first phase of observation, fibrin from individuals was studied from the menstrual phase (days 1-5). Figures $1 \mathrm{~A}-\mathrm{D}$ shows different magnification from this phase. It is important to notice that the highest magnification (machine magnification $150,000 \times)$ showed smooth fibrin fibers.

Figures 2A-D shows fibrin networks from the preovulatory phase (days 12-14 of the menstrual cycle). During this phase, the fibers do not appear smooth, and it seems as if the fibrinogen, on activation with thrombin, causes a globular placement of the molecules that form the resulting fibrin fibers. Alternatively, the fibrin fibers might be stickier, attracting free plasma proteins to it, which stick to the individual fibers to form this globular ultrastructure. Also, a more flimsy fiber morphology is noted in Figure 2A (if compared to Fig. 1A)-which give a typical lower magnification view of the fibrin network.

The final phase of analysis was during the luteal phase (days 20-25) of the menstrual cycle. Figures $3 \mathrm{~A}-\mathrm{D}$ shows micrographs form this phase. The fibers appear more flimsy (compare 1A, 2A, and 3A) and also have globular deposits (Fig. 3D). During this phase, the minor, thin fibers are more prominent between the thick fibers if compared to the first phase (Fig. 1B). Here, it also seems as if the individual fibers are fused possibly because of a more sticky nature.

Figure 4 displays different angles of sections through the fibrin fibers. In Figure $4 \mathrm{~A}$ the fibrin internal structure during the menstrual phase (days 1-5) is shown, while Figures 4B-D shows the internal morphology of the fibrin fibers during the preovulatory (days 12-14) and luteal (days 20-25) phases. The external and internal structure of the fibrin network appears smooth during the menstrual phase (Fig. 4A). Globules similar in shape and size to those seen on the external fibrin network structure (Figs. 2C,D) can be distinguished. Some globules appear to be on the surface of the fibrin strand (Fig. 4B, black arrow), while others form part of the fiber (Fig. 4C, white arrow). Some dark, globular clusters can also be distinguished within the lighter fibrin fibers (Fig. 4D, gray arrows).

Estradiol Concentrations. All participants exhibited estradiol levels within the normal ranges as specified by Ampath pathology laboratory support services. These levels are as follows: follicular phase (coincides with menstrual phase of days 1-5 of the cycle) ranged between 99 and $448 \mathrm{pmol} / \mathrm{L}$; mid-cycle 

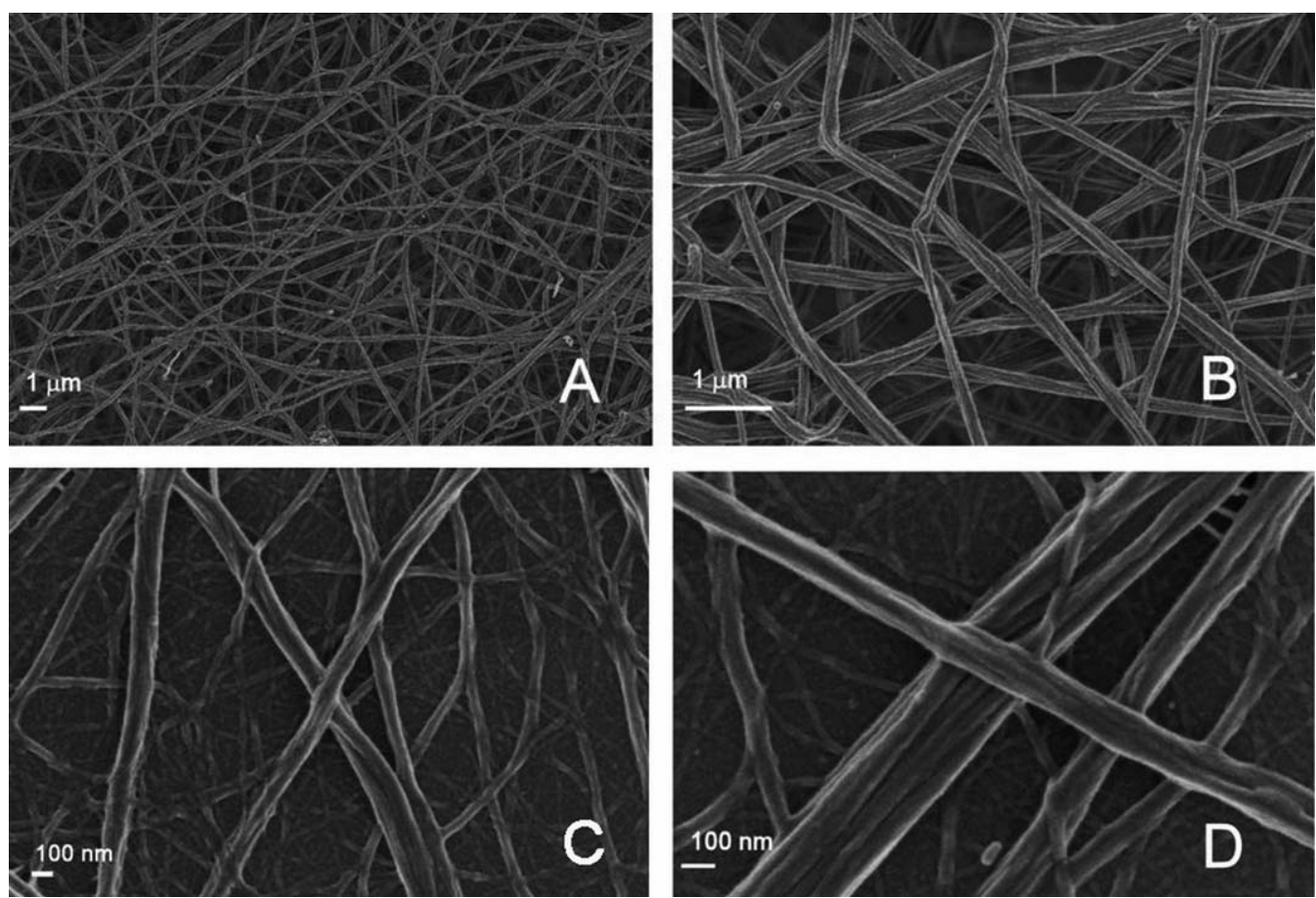

Fig. 1. Scanning electron micrographs of fibrin networks observed during the menstrual phase (days 1-5) of the menstrual cycle. Machine magnification: (A) $10,000 \times$, (B) $40,000 \times$, (C) $100,000 \times$, (D) $150,000 \times$.
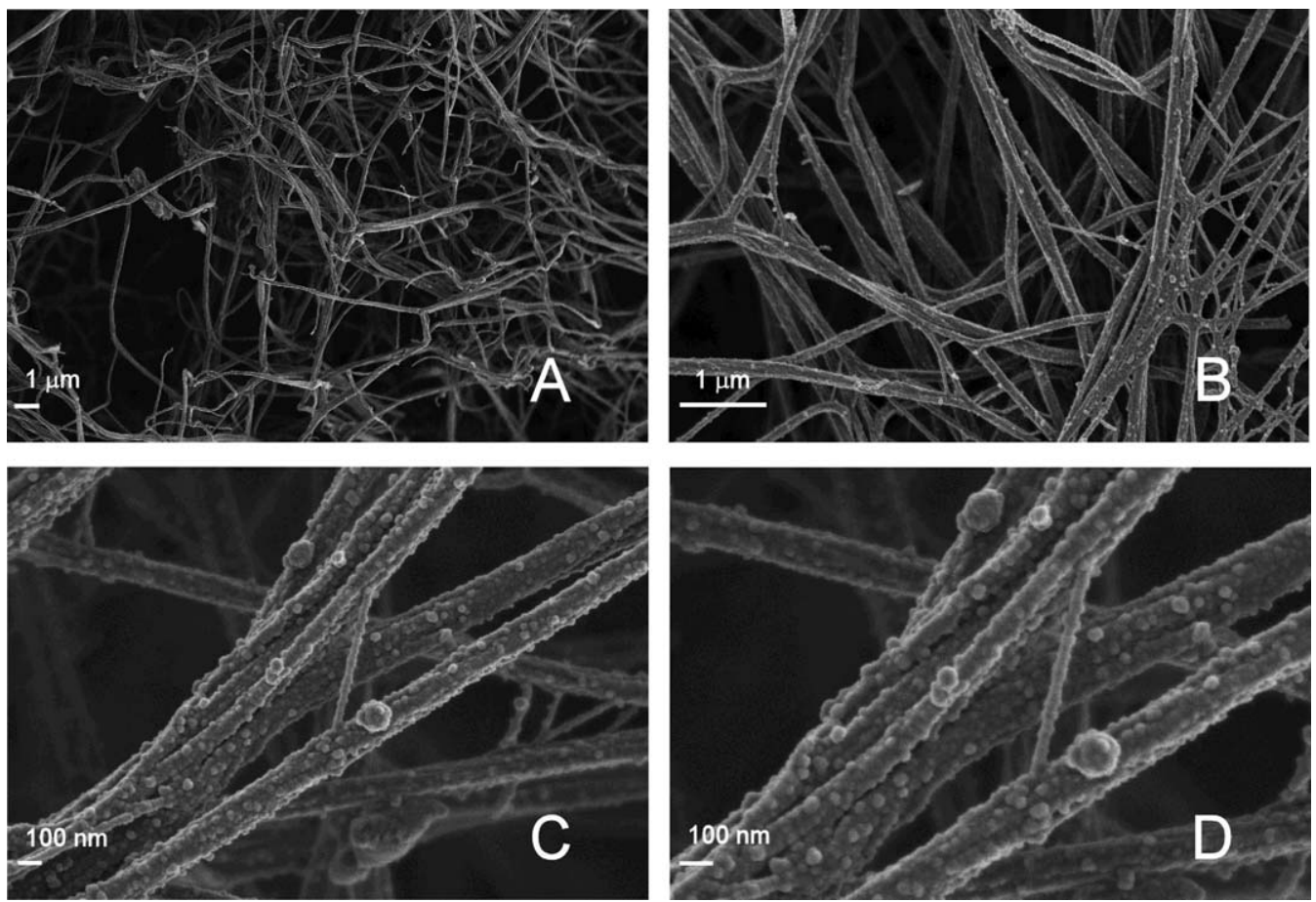

Fig. 2. Scanning electron micrographs of fibrin networks observed during the period leading up to ovulation (days 12-14) of the menstrual cycle. Machine magnification: (A) $10,000 \times$, (B) $40,000 \times$, (C) $100,000 \times$, (D) $150,000 \times$ 

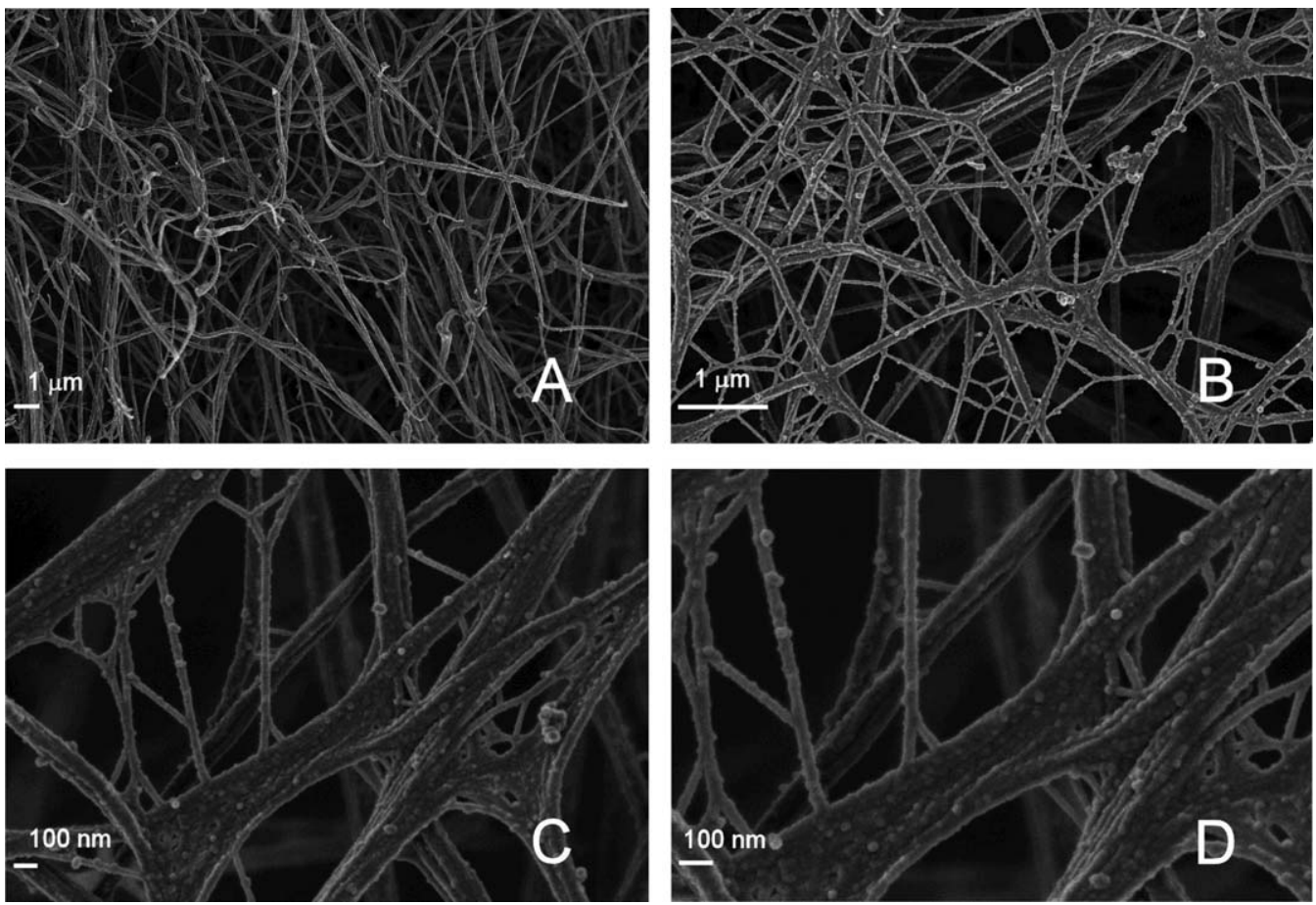

Fig. 3. Scanning electron micrographs of fibrin networks observed during the luteal phase (days 20 25) of the menstrual cycle. Machine magnification: (A) $10,000 \times$, (B) $40,000 \times$, (C) $100,000 \times$, (D) $150,000 x$.

(coincides with pre-ovulatory phase of days 12-14 of the cycle) ranged between 180 and $1068 \mathrm{pmol} / \mathrm{L}$; luteal phase (days 20-25 of the cycle) ranged between 349 to 1,589 pmol/L. These values are shown in Table 1.

The average observed for the participants were as follows: $125 \mathrm{pmol} / \mathrm{L}$ for the menstrual phase, $751 \mathrm{pmol} /$ $\mathrm{L}$ for the pre-ovulatory phase, and $423 \mathrm{pmol} / \mathrm{L}$ for the luteal phase. These values are also shown in Table 1.

\section{Experiment 2}

Since the alterations in fibrin morphology seen in Experiment 1 and 2 coincided with the estrogen peaks in the menstrual cycle, estrogen was added to a fibrinogen model to determine the exclusive effect of estrogen on the fibrin network.

A normal fibrin network is shown in Figure 5A, where a network of smooth fibers is shown.

In Figure 5B, the effect of exclusively estrogen on the fibrin network can be seen. The fibers also have globular plasma protein deposits on the formed fibrin fibers, similar to the fibers in Figures 2 and 3.

\section{DISCUSSION}

Haemostasis is the dynamic balance maintained between the coagulation system and the fibrinolytic system (Knol et al., 2012) and it is well known that, in females, there are cyclical variability in estradiol and progesterone during the follicular and luteal phases (Trigg et al., 2011). Although the hormonal changes during the cycle are a well-known phenomenon, the question that arises is whether coagulation plasma protein concentrations vary significantly, and how hormonal changes influence this. Knol et al. (2012) attempted to answer this by systematically reviewing literature discussing variations and those reports suggesting no variation in coagulation protein concentrations during the different phases of the female cycle. From their review, they concluded that the optimal timing of haemostatic testing seems to be the menstrual and early follicular phase of the menstrual cycle (Knol et al., 2012).

Some interesting results of variations in haemostatic variability include D-dimer differences during the follicular and luteal phases and as estradiol concentration increased, PAI-I decreased. D-dimer is a fibrin degradation product or a small protein fragment present in the blood after a blood clot is degraded by fibrinolysis. PAI is a serine protease inhibitor that is the principal inhibitor of tissue plasminogen activator (tPA) and urokinase (uPA), the activators of plasminogen and ultimately, fibrinolysis. During the menstrual cycle, changes have also been observed in levels of von Willebrand factor (VWF), fibrinogen, and activated factor VII (Trigg et al., 2011). Kadir et al. (1999) mentioned that in healthy individuals, fibrinogen, von Willebrand factor antigen and von Willebrand factor activity concentrations showed strong cyclic variations with peak values in the luteal phase (Kadir et al., 1999). Knol et al. (2012) reported that VWF-levels were mostly about $10 \%$ (range $2-24 \%$ ) lower in menstrual/early follicular phase compared to the luteal phase. However, the authors found conflicting literature regarding the levels of fibrinogen during the menstrual cycle. The main constant factor of change during the menstrual cycle is therefore estrogen levels and it can be concluded that estrogen has an influence 

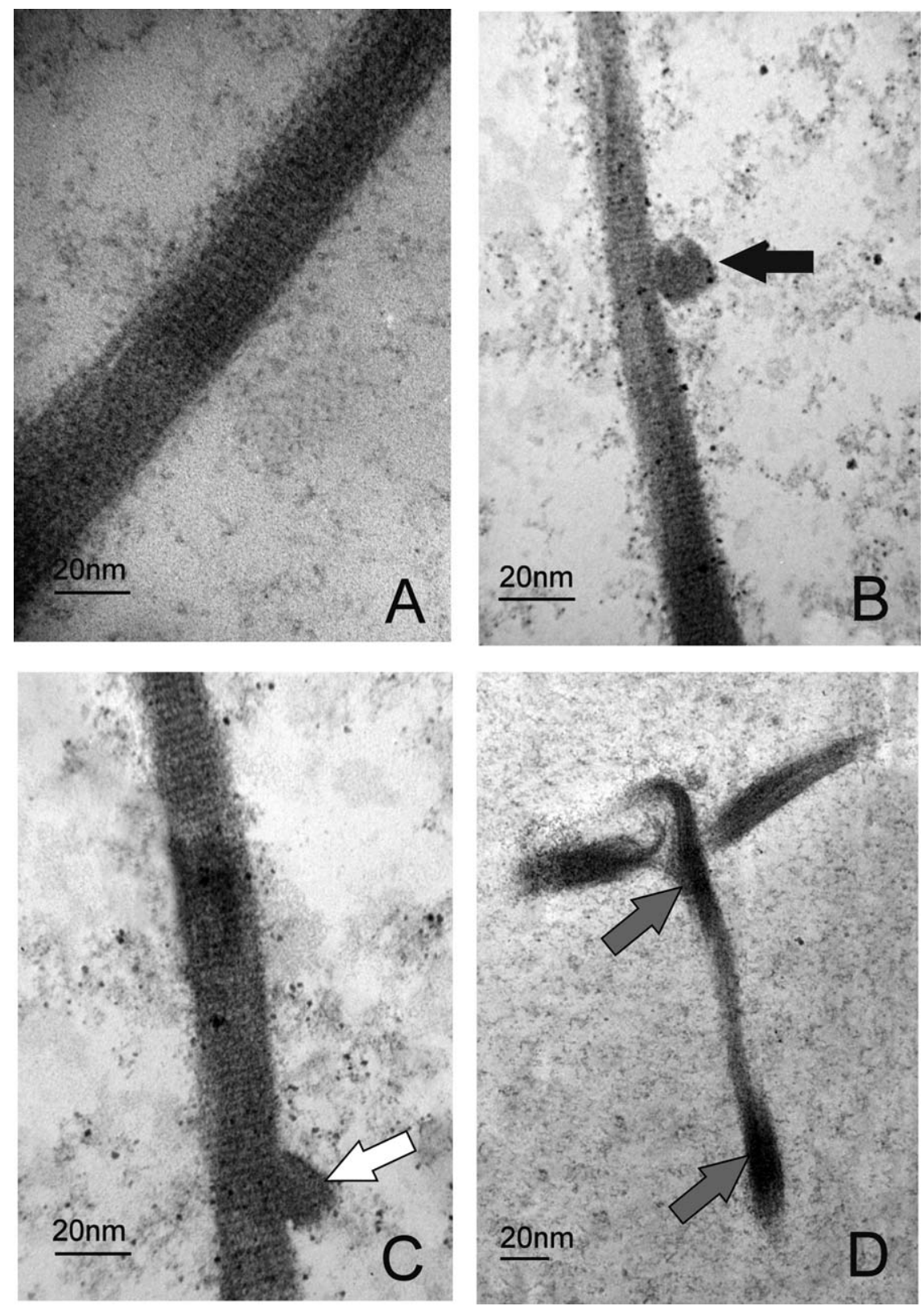

Fig. 4. Transmission electron micrographs of fibrin networks observed during the menstrual cycle. A: Menstrual phase (days 1-5), $\mathrm{B}-\mathrm{D}$ pre-ovulatory (days $12-14$ ) and luteal (days $20-25$ ) phases of the

menstrual cycle. Black arrow $=$ Globules on the surface of the fibrin fiber (B), White arrow $=$ Globules forming part of the fibrin fiber $(C)$, Gray arrows $=$ dark globular clusters within the fibrin fibers (D).

on the plasma proteins and their packaging during fibrin fiber network formation (Knol et al., 2012).

Therefore, during the menstrual cycle of healthy individuals, the ultrastructural profiles of fibrin networks were analyzed by following a qualitative analysis of fibrin fibers. In the current research, we found that the fibrin fibers are smooth during days $1-5$ of the menstrual cycle. However, during days $12-14$, the fibrin fiber morphology start to change (Figs. 2A-D), becoming less smooth, and during the luteal phase of the cycle (days 20-25), the network appears sticky, where the minor, thin fibers are more prominent

between the thick fibers (Fig. 3B) if compared to the first phase (Fig. 1B).

These changes noted in the fibrin networks were confirmed to be due to changes in levels of estrogen. As mentioned previously, there are two estrogen peaks, the first estrogen peak is between day 12 and 14 and then also during the luteal phase, a second peak of estrogen occurs (Mosesson, 2005). These peaks coincide with the changes we see in the current qualitative research, where the fibrin morphology changes during the same time as the estrogen peaks occur. The effect of estrogen on the fibrin network in the menstrual 
TABLE 1. Estradiol concentrations

\begin{tabular}{lccc}
\hline & $\begin{array}{c}\text { Menstrual } \\
\text { phase }\end{array}$ & $\begin{array}{c}\text { Pre-ovulatory } \\
\text { phase }\end{array}$ & $\begin{array}{c}\text { Luteal } \\
\text { phase }\end{array}$ \\
\hline Ampath specified ranges & $99-448$ & $180-1,068$ & $349-1,589$ \\
Averages for participants & 125 & 751 & 423 \\
\hline
\end{tabular}

Ranges for specific periods in the menstrual cycle as specified by Ampath pathology laboratory support services and averages for participants. Values given as estradiol concentration in $\mathrm{pmol} / \mathrm{L}$.

cycle was also confirmed with a fibrinogen model, where the addition of estrogen altered the fibrin network morphology in the same manner as seen in whole blood.

Investigation of the internal structure of the fibrin fibers confirmed the presence of globules on the fibrin network. These globules were not only present on the surface of the fibrin fiber strand, but some of these globules formed part of the strand itself. This shows that the altered morphology is not only confined to the outer surface of the fibers. Since dark globular clusters also appeared on the inside of the fibrin fibers, it can be assumed that the formation of the fibers is influenced by estrogen, not only the final structure.

The globules present on and within the fibrin network are thought to be due to a change in the packaging of the fibrinogen, as Knol et al. (2012) stated.

These changes in fibrin ultrastructure may have a dual function. First, it fertilization takes place this altered fibrin fibers may support the implantation of a fertilized ovum. Secondly, if fertilization does not take place, this changed morphology will ensure sufficient coagulation during menses to prevent excessive blood loss.

Some limitations exist for this study. Since these findings are only ultrastructural analysis of the fibrin network, biochemical analysis of the altered morphology may indicate the mechanisms involved in these unique results. It may shed some more light on the origin of the globules seen on and within the fibrin fibers.

Our research group has studied and compiled a database of healthy individuals with 1000's of fibrin network micrographs. Since the micrographs from the six participants compared well with our database and the same trends were seen for all the participants we expect the same results for additional participants.
For further investigation, a larger sample size could be employed; however we believe that the results will remain the same for a larger participant group.

Estrogen have shown neuroprotective properties during acute CNS trauma and stroke (Roof and Hall, 2000), lung injury induced by trauma (Kan et al., 2008) and a cardioprotective role following trauma-hemorrhage (Yu et al., 2007). It would therefore be of great value to investigate possible differences between the fibrin network formation of males and females as well as the effect of estrogen on male plasma.

Gender plays a role in coagulability and clot strength. Thromboelastography (TEG) analysis of male and female blood has revealed that females show significantly increased hypercoagulability when compared to males. The initial rate of fibrin formation along with the rate of clot growth was shown to be much faster and higher in females than in males. The maximal clot strength and the viscoelastic properties of the formed clot were also increased in women. These changes were attributed to quicker thrombin generation and greater velocity in women. The value for total thrombus generation (TTG) was also significantly increased in females (Roeloffzen et al., 2010). Fibrinogen and Factor XIII concentrations have been correlated with increased clot strength (Nielsen et al., 2004; Schroeder et al., 2001; Tyler, 1969). Females have much higher levels of fibrinogen (Eliasson et al., 1993) and factor XIII (Ariëns et al., 1999), the enzyme responsible for the crosslink of fibrin, than men. The increased clot strength and changes in fibrin morphology brought on by female hormones such as estrogen may be the contributing factors responsible for increased hypercoagulability and risk of venous thromboembolism in women during pregnancy and with the use of oral contraceptives.

It is therefore of great importance to investigate the effect of female hormones on coagulation in more depth. It is clear that hormones such as estrogen influence the ultrastructure of fibrin networks. The effect of other hormones, such as progesterone and testosterone, separately and in conjunction with estrogen will need to be investigated to provide more detail into the effect of sexual hormones on coagulation.
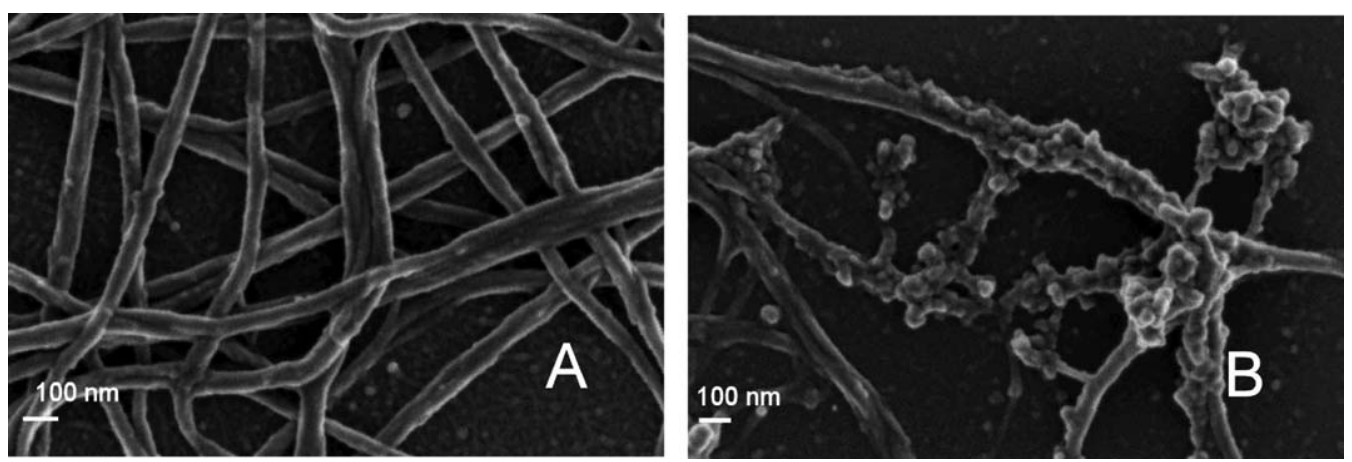

Fig. 5. Scanning electron microscopy analysis of fibrin networks from a fibrinogen model with added estrogen. A: Fibrinogen without the addition of estrogen. B: Fibrinogen and estrogen (pre-ovulatory concentration). Machine magnification: $150,000 \times$. 


\section{CONCLUSION}

During the menstrual cycle, the fibrin fiber morphology differs. Estrogen causes the changes in the external and internal structure of the fibrin networks identified during the menstrual cycle. This research therefore is the first to show ultrastructural changes in fibrin fiber morphology resulting from the estrogen changes during the menstrual cycle.

In the second part of this research article in this issue, namely Ultrastructural changes of fibrin networks during three phases of pregnancy: a qualitative investigation, similar alterations as shown in this manuscript are shown during different phases of pregnancy. These changes in fibrin network ultrastructure associated with elevated estrogen levels may possibly shed light on pathological mechanisms employed in the development of abnormal or ailing pregnancy

\section{ETHICAL CONSIDERATIONS}

Ethical clearance was obtained from the Human Ethics Committee from the University of Pretoria. All individuals completed informed consent forms.

\section{ACKNOWLEDGMENTS}

The authors acknowledge Mrs. M.C. van Zijl for her assistance with the estrogen concentrations. Also S. van Wyk for her assistance in the blood collections.

\section{REFERENCES}

Ariëns RAS, Kohler HP, Mansfield MW, Grant PW. 1999. Subunit antigen and activity levels of blood coagulation factor XIII in healthy individuals: Relation to sex, age, smoking, and hypertension. Arterioscler Thromb Vasc Biol 19:2012-2016.

Bailey AL, Scantlebury DC, Smyth SS. 2009. Thrombosis and antithrombotic therapy in women. Arterioscler Thromb Vasc Biol 29:284288.

Butenas S, Mann K. 2002. Blood coagulation. Biochemistry (Moscow) 67:3-12.

Dighe AS, Moy JM, Hayes FJ, Sluss PM. 2005. High-resolution reference ranges for estradiol, luteinizing hormone, and folliclestimulating hormone in men and women using the AxSYM assay system. Clin Biochem 38:175-179.

Eliasson M, Evrinb PE, Lundblada D, Asplundc K, Rånbyd M. 1993. Influence of gender, age and sampling time on plasma fibrinolytic variables and fibrinogen: A population study. Fibrinolysis 7:316 323.

Giardina E-GV, Chen HJ, Sciacca RR, Rabbani LE. 2004. Dynamic variability of hemostatic and fibrinolytic factors in young women. J Clin Endocrinol Metab 89:6179-6184.

Herd C, Page C. 1994. Pulmonary immune cells in health and disease: Platelets. Eur Respir J 7:1145-1160.

Kadir RA, Economides DL, Sabin CA, Owens D, Lee CA. 1999. Variations in coagulation factors in women: Effects of age, ethnicity, menstrual cycle and combined oral contraceptive. Thromb Haemost 82:1456-1461.

Kan WH, Hsu JT, Schwacha MG, Choudhry, MA, Bland KI, Chaudry IH. 2008. Estrogen ameliorates trauma-hemorrhage-induced lung injury via endothelial nitric oxide synthase-dependent activation of protein kinase G. Ann Surg 248:294-302.

Knobil E, Neill JD, Adashi EY. 1998. Encyclopedia of reproduction. San Diego: Academic Press.

Leng X-H, Hong SY, Larrucea S, Zhang W, Li T-T, López JA, Bray PF 2004. Platelets of female mice are intrinsically more sensitive to agonists than are platelets of males. Arterioscler Thromb Vasc Biol 24:376-381.

Liao S-L, Chen W-Y, Kuo J-S, Chen C-J. 2001. Association of serum estrogen level and ischemic neuroprotection in female rats. Neurosci Lett 297:159-162.

Lowe GD, Rumley A, Mackie IJ. 2004. Plasma fibrinogen. Ann Clin Biochem 41:430-440.

Knol HM, Kemperman RFJ, Kluin-Nelemans HC, Mulder AB, Meijer K. 2012. Haemostatic variables during normal menstrual cycle: A systematic review. Thromb Haemost 107:22-29.

Mosesson M. 2005. Fibrinogen and fibrin structure and functions. J Thromb Haemost 3:1894-1904.

Nielsen VG, Gurley WQ Jr, Burch TM. 2004. The impact of factor XIII on coagulation kinetics and clot strength determined by thrombelastography. Anesth Analg 99:120-123.

Niswender G, Nett T. 1994. Corpus luteum and its control in infraprimate species. Physiol Reprod 1:781-816.

O'Riordan MN, Higgins JR. 2003. Haemostasis in normal and abnormal pregnancy. Best Pract Res Clin Obstet Gynaecol 17:385-396.

Roeloffzen WWH, Kluin-Nelemans HC, Mulder AB, Veeger NJGM Bosman L, de Wolf JTM. 2010. In normal controls, both age and gender affect coagulability as measured by thrombelastography. Anesth Analg 110:987-994.

Roof RL, Hall ED. 2000. Gender differences in acute CNS trauma and stroke: neuroprotective effects of estrogen and progesterone. J Neurotrauma 17:367-388.

Schroeder V, Chatterjee T, Kohler H. 2001. Influence of blood coagulation factor XIII and FXIII val34leu on plasma clot formation measured by thrombelastography. Thromb Res 104:467-474.

Silverthorn DU, Ober WC, Garrison CW, Silverthorn AC, Johnson BR. 2009. Human physiology: An integrated approach. Cummings: Pearson/Benjamin.

Suzuki S, Brown CM, Wise PM. 2009. Neuroprotective effects of estrogens following ischemic stroke. Front Neuroendocrinol 30: 201-211.

Trigg DE, Wood MG, Kouides PA, Kadir RA, Cordle E, Govind A, Lu Y, Wang S, Sullivan-Halley J, Chang E. 2011. Hormonal influences on hemostasis in women. Sem Thromb Hemost 37:77-86.

Tyler HM. 1969. Fibrin crosslinking demonstrated by thrombelastography. Thromb Diath Haemorrh 22:398-400.

van der Spuy WJ, Pretorius E. 2012. Interrelation between inflammation, thrombosis, and neuroprotection in cerebral ischemia. Rev Neurosci 23:269-278.

Wong JH, Dukes J, Levy RE, Sos B, Mason SE, Fong TS, Weiss EJ. 2008. Sex differences in thrombosis in mice are mediated by sexspecific growth hormone secretion patterns. J Clin Investig 118 2969.

Yu HP, Hsieh YC, Suzuki T, Choudhry MA, Schwacha MG, Bland KI, Chaudry IH. 2007. The PI3K/Akt pathway mediates the nongenomic cardioprotective effects of estrogen following trauma-hemorrhage. Ann Surg 245:971-977. 